\title{
Clear Cell adenocarcinoma of THE ReNAl Pelvis: AN EXTREMELY RARE NEOPLASM OF THE UPPER URINARY TRACT
}

\author{
Kai-Wen LiU ${ }^{1,2}$, Victor Chia-Hsiang Lin ${ }^{3,4}$, I-Wei Chang $^{1,4}$
}

${ }^{1}$ Department of Pathology, E-DA Hospital, Kaohsiung, Taiwan

${ }^{2}$ Department of Pathology, Kaohsiung Chang Gung Memorial Hospital and Chang Gung University College of Medicine, Kaohsiung, Taiwan

${ }^{3}$ Department of Urology, E-DA Hospital, Kaohsiung, Taiwan

${ }^{4}$ Institute of Biotechnology and Chemical Engineering, I-Shou University, Kaohsiung, Taiwan

Clear cell adenocarcinoma (CCA) in the urinary tract is a rare neoplasm morphologically identical to the Müllerian counterpart. Clear cell adenocarcinoma is extremely rare in the upper urinary tract. We present a case with CCA of the renal pelvis. Microscopically, the tumor exhibited exophytic growth with predominantly tubulocystic structures, as well as solid and papillary patterns. The neoplastic cells were cuboidal with clear to pale eosinophilic cytoplasm and abundant intracellular and extracellular eosinophilic hyaline globules. By immunohistochemically, the tumor was labeled by cytokeratins and hepatocyte nuclear factor- $1 \beta$. The patient was still alive without evidence of recurrence in the follow-up period of nineteen months after diagnosis.

Key words: clear cell adenocarcinoma, hepatocyte nuclear factor- $1 \beta$, renal pelvis, upper urinary tract.

\section{Introduction}

Clear cell adenocarcinoma (CCA) usually occurs in the female genital tract - vagina, uterine cervix, endometrium and ovary, as a distinct entity. In the uri-

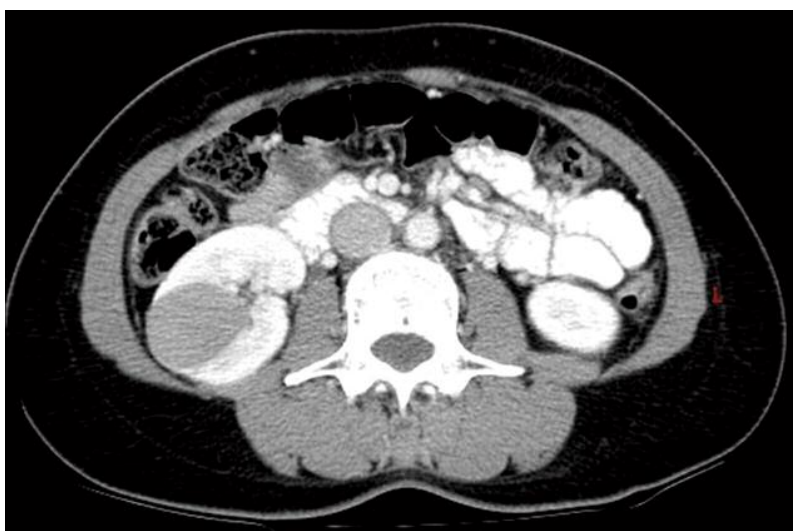

Fig. 1. Abdominal computed tomography scan showed a well-defined tumor, measuring $4.3 \mathrm{~cm} \times 3.8 \mathrm{~cm}$, at the right kidney, with effacement of middle and lower calyces nary system, CCA is a rare neoplasm resembling its Müllerian counterpart and usually found in the lower urinary tract, predominantly the urethra, of women $[1,2]$. In contrast to the poor prognosis of CCA of the female genital tract, CCA of the lower urinary tract is not as aggressive as initially believed. Many of these tumors have an exophytic growth pattern; therefore, they may be diagnosed at an early stage and have a relatively good prognosis [3]. Clear cell adenocarcinoma arising in the upper urinary tract is extremely rare. To the best of our knowledge, only one case has been reported in the English literature [4]. Herein, this report describes a CCA of the renal pelvis, which is the second documented case of this distinct variant of urinary tract neoplasms in the upper urinary tract.

\section{Case report}

A 43-year-old woman presented to the hospital with a complaint of intermittent episodes of painless gross hematuria for one month. Computed tomography (CT) 

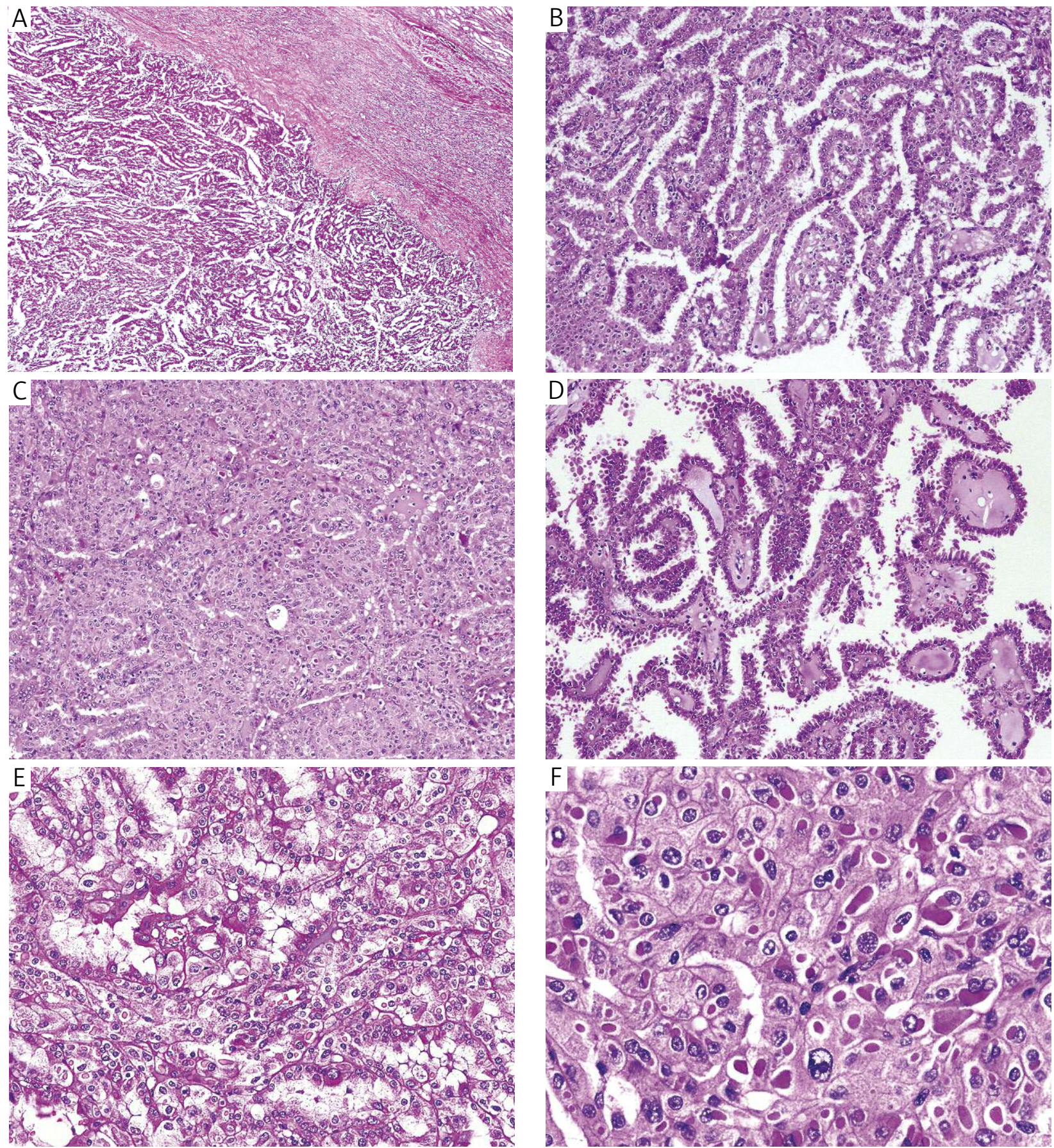

Fig. 2. Histopathological features. Low power view showed an exophytic tumor without subepithelial stromal invasion (A). The tumor exhibited tubulocystic (B), solid (C) and papillary growth patterns (D). Note the hobnail-like lining cells of the papillary fronds (D). High power view revealed cuboidal neoplastic cells with clear to pale eosinophilic cytoplasm (E) and abundant intracellular and extracellular eosinophilic hyaline globules (F). (A: HE, 20×; B-D: HE, 100×; E: HE, 200×; F: HE, 400×)

of the abdomen demonstrated a well-defined mass measuring $6.0 \mathrm{~cm} \times 4.3 \mathrm{~cm}$ at the right kidney with effacement of middle and lower calyces (Fig. 1). The patient received surgical intervention of right nephroureterectomy for treatment. She also has been followed up clinically as well as by serial CT imaging of the abdomen. She was alive without evidence of recurrence 19 months after surgery.

\section{Pathological findings}

The received right kidney measured $10.0 \mathrm{~cm} \times 7.0 \mathrm{~cm}$ $\times 6.0 \mathrm{~cm}$ and weighed $140 \mathrm{~g}$. On the cut section, a protruding, fragile, yellowish-white mass, measuring $6.5 \mathrm{~cm}$ $\times 5.5 \mathrm{~cm} \times 5.4 \mathrm{~cm}$, was noted occupying the renal pelvis and compressing the renal parenchyma. Histological examination showed an exophytic tumor arising 

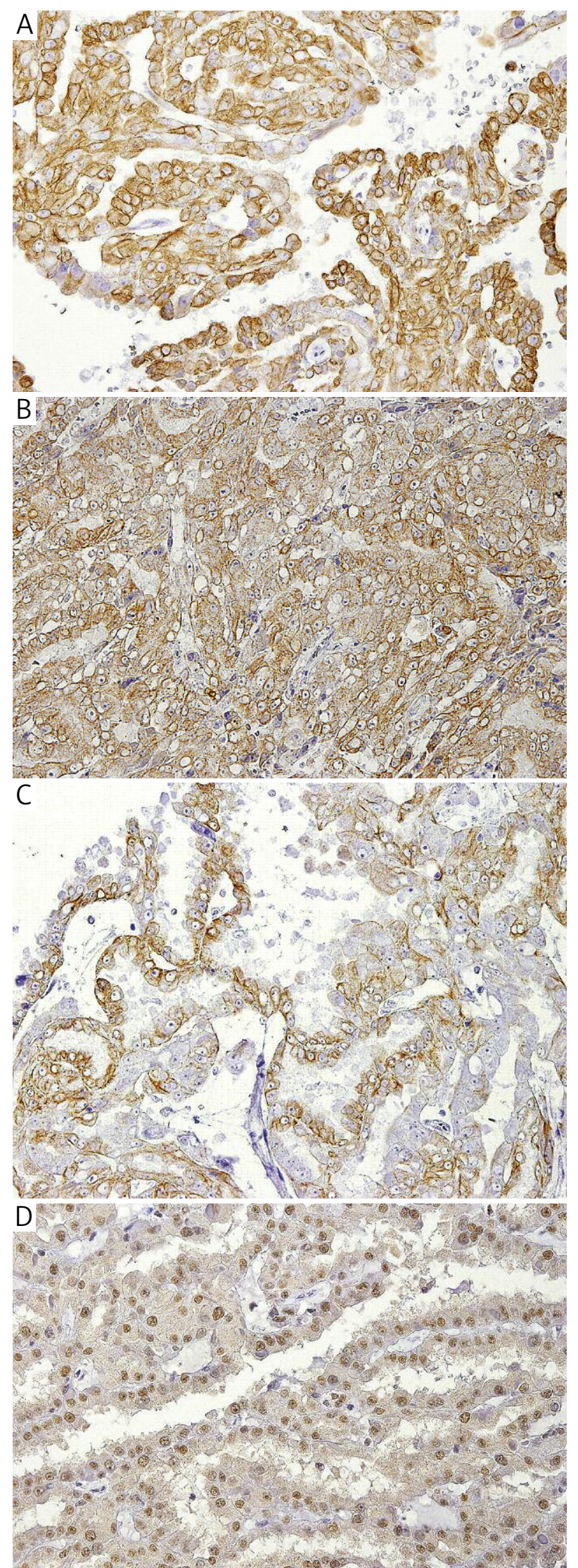

Fig. 3. Immunohistochemical study. The tumor cells were immunoreactive to CK7 (A), low- (B) and high-molecularweight cytokeratins $(C)$, as well as hepatocyte nuclear factor-1 $1 \beta$ (D). (A-D: immunostains, 200×) from the renal pelvis without subepithelial stromal invasion (Fig. 2A). The tumor was composed predominantly of tubulocystic structures (Fig. 2B) while solid areas (Fig. 2C) and papillary structures (Fig. 2D) were also seen. The neoplastic cells were cuboidal with clear to pale eosinophilic cytoplasm (Fig. 2E) and abundant intracellular and extracellular eosinophilic hyaline globules (Fig. 2F). Moderate cytological atypia, irregular nuclear membrane and prominent nucleoli were also observed (Fig. 2F). Hobnail-like cells were occasionally seen (Fig. 2D). Neither regional lymph node nor concurrent urothelial carcinoma was identified. The pathological stage for the right renal pelvic tumor was pTaNOMX according to the latest American Joint Committee on Cancer (AJCC) Cancer Staging Manual [5].

Immunohistochemically, the tumor cells were strongly immunoreactive to cytokeratin 7 (Fig. 3A), low-molecular-weight cytokeratin (Fig. 3B), high-molecular-weight cytokeratin (Fig. 3C) and hepatocyte nuclear factor (HNF)-1 $\beta$ (Fig. 3D).

\section{Discussion}

Clear cell adenocarcinoma in the urinary tract is a rare neoplasm predominantly occurring in the lower urinary tract, especially the urethra, of females. This distinct neoplasm is morphologically identical to that of the same name that arises in female genital organs. The histogenesis of CCA of the urinary tract has remained controversial. The four prevailing theories includef mesonephric origin, Müllerian origin, urothelial origin and malignant transformation of nephrogenic adenoma. Clear cell adenocarcinoma of the urinary tract was initially assumed to be of mesonephric origin and was designated as mesonephric carcinoma [6]. A possible renal tubular cell differentiation/mesonephric origin was also described by some authors due to the immunohistochemical profile in their study [7]. Because of the histological similarities with the homonymous tumor of the female genital tract and the marked predominance of CCA of the lower urinary tract as well as the associated endometriosis and Müllerian duct remnants in some cases, the tumor was considered to be of Müllerian derivation by some authors $[3,8,9]$. Clear cell adenocarcinoma was also postulated to be of urothelial origin. In a recent study [10], CCAs exhibited genetic aberrations identical to those commonly observed in urothelial carcinoma by fluorescence in situ hybridization assays. A common clonal origin was also proved in two cases with concurrent CCA and urothelial carcinoma by $\mathrm{X}$-chromosome inactivation analysis [10]. Finally, nephrogenic adenoma is an important differential diagnosis of CCA. Occasional cases with morphological or molecular evidence for progression of nephrogenic adenoma of the urinary bladder to CCA have been reported $[11,12]$.

Clear cell adenocarcinoma is extremely rare in the upper urinary system. There has been only one case re- 
ported of CCA arising from the upper ureter and renal pelvis in the English literature [4]. Unlike the relatively favorable clinical outcome of the present case, this 73-year-old woman suffered from multiple pulmonary metastases and eventually died of the disease five months later. In contrast, the present patient was still alive without evidence of disease at 19-month follow-up. The discrepancy between the different clinical behaviors of the same tumor type arising in the identical anatomical location may be due to the low tumor stage (pTaN0MX) of the present case. To the best of our knowledge, this report documents the second case of CCA in the upper urinary tract, and also the first case of CCA confined to the renal pelvis.

Hepatocyte nuclear factor (HNF)- $1 \beta$ is a transcription factor that is normally expressed in the liver, gastrointestinal tract, pancreas, and kidney, and is involved in the regulation of glucose homeostasis, glycogen accumulation, and anti-apoptosis [13]. Up-regulation of HNF-1 $\beta$ was first identified in ovarian CCAs in terms of both mRNA and protein level [14]. After that, HNF-1 $\beta$ was also investigated as a sensitive and specific marker for both ovarian and endometrial CCAs, whereas other subtypes of tumors arising in these regions rarely expressed this marker by immunohistochemical study $[14,15]$. Recently, HNF-1 $\beta$ has also been investigated as a useful marker in differentiating CCAs of the bladder/urethra from urothelial carcinoma and other types of bladder adenocarcinoma [2]. In this study, all 18 CCAs exhibited nuclear staining in more than $90 \%$ of tumor cells. This specific finding identical to gynecologic CCAs may point to a Müllerian derivation/differentiation. To our knowledge, our study documents the first upper urinary tract CCA showing HNF-1 $\beta$ immunostaining. However, from a differential diagnosis standpoint, HNF- $1 \beta$ may only serve as a marker to differentiate CCA from other primary tumors of the urinary tract, but not metastatic CCA from the female genital tract or primary clear cell renal cell carcinoma [16].

In conclusion, we have reported a rare case of primary CCA, which is the first documented case confined to the renal pelvis. The histomorphology and immunohistochemical features of our case were identical to the female genital tract and lower urinary tract counterparts. HNF-1 $\beta$, a sensitive and fairly specific marker for CCAs of the female genital tract and lower urinary tract, may be useful for the diagnosis of the same type of neoplasm occurring in the upper urinary tracts.

The authors declare no conflict of interests.

This work was partially supported by grants from $E-D A$ Hospital (grant number EDAHP102003).

\section{References}

1. Oliva E, Young RH. Clear cell adenocarcinoma of the urethra: a clinicopathologic analysis of 19 cases. Mod Pathol 1996; 9: 513-520.

2. Brimo F, Herawi M, Sharma R, et al. Hepatocyte nuclear factor-1beta expression in clear cell adenocarcinomas of the bladder and urethra: diagnostic utility and implications for histogenesis. Hum Pathol 2011; 42: 1613-1619.

3. Drew PA, Murphy WM, Civantos F, Speights VO. The histogenesis of clear cell adenocarcinoma of the lower urinary tract. Case series and review of the literature. Hum Pathol 1996; 27 : 248-252.

4. Shih CM, Huang CT, Chi CH, et al. CA125-producing clear cell adenocarcinoma arising from the upper ureter and renal pelvis. J Chin Med Assoc 2010; 73: 40-43.

5. Edge SB, Byrd DR, Compton CC, et al. (eds.). AJCC cancer staging manual. $7^{\text {th }}$ ed. Springer-Verlag, New York 2009; 491-496.

6. Konnak JW. Mesonephric carcinoma involving the urethra. J Urol 1973; 110: 76-78.

7. Alexiev BA, Tavora F. Histology and immunohistochemistry of clear cell adenocarcinoma of the urethra: histogenesis and diagnostic problems. Virchows Arch 2013; 462: 193-201.

8. Chor PJ, Gaum LD, Young RH. Clear cell adenocarcinoma of the urinary bladder: report of a case of probable mullerian origin. Mod Pathol 1993; 6: 225-228.

9. Mai KT, Yazdi HM, Perkins DG, et al. Multicentric clear cell adenocarcinoma in the urinary bladder and the urethral diverticulum: evidence of origin of clear cell adenocarcinoma of the female lower urinary tract from Müllerian duct remnants. Histopathology 2000; 36: 380-382.

10. Sung MT, Zhang S, MacLennan GT, et al. Histogenesis of clear cell adenocarcinoma in the urinary tract: evidence of urothelial origin. Clin Cancer Res 2008; 14: 1947-1955.

11. Hartmann A, Junker K, Dietmaier W, et al. Molecular evidence for progression of nephrogenic metaplasia of the urinary bladder to clear cell adenocarcinoma. Hum Pathol 2006; 37: 117 120.

12. Suttmann H, Holl-Ulrich K, Peter M, et al. Mesonephroid adenocarcinoma arising from mesonephroid metaplasia of the urinary bladder. Urology 2006; 67: 846.e7-8.

13. Senkel S, Lucas B, Klein-Hitpass L, Ryffel GU. Identification of target genes of the transcription factor HNF1beta and HNF1alpha in a human embryonic kidney cell line. Biochim Biophys Acta 2005; 1731: 179-190.

14. Tsuchiya A, Sakamoto M, Yasuda J, et al. Expression profiling in ovarian clear cell carcinoma: identification of hepatocyte nuclear factor-1 beta as a molecular marker and a possible molecular target for therapy of ovarian clear cell carcinoma. Am J Pathol 2003; 163: 2503-2512.

15. Yamamoto S, Tsuda H, Aida S, et al. Immunohistochemical detection of hepatocyte nuclear factor 1beta in ovarian and endometrial clear-cell adenocarcinomas and nonneoplastic endometrium. Hum Pathol 2007; 38: 1074-1080.

16. Wang CC, Mao TL, Yang WC, Jeng YM. Underexpression of hepatocyte nuclear factor-1beta in chromophobe renal cell carcinoma. Histopathology 2013; 62: 589-594.

\section{Address for correspondence}

\section{I-Wei Chang, MD}

Department of Pathology, E-DA Hospital

No. 1, Yi-Da Road, Jiao-Su Village, Yan-Chao District

Kaohsiung City 824, Taiwan

tel. +886-7-615-0011 ext. 2620

fax +886-7-615-0974

e-mail: ed106242@edah.org.tw 